\title{
Association between the expression of inhibitor of growth family member 4 and the progression of clear cell renal carcinoma
}

\author{
YUXIN REN $^{1}$, SONG ZHAO $^{2}, \mathrm{HE} \mathrm{CHEN}^{3}$, YING-MEI FU $^{4}$ and BAI ZHAO ${ }^{5}$ \\ Departments of ${ }^{1}$ Oncology and ${ }^{2}$ General Surgery, The First Affiliated Hospital of Harbin Medical University, Harbin, \\ Heilongjiang 150001; Departments of ${ }^{3}$ Pathology and ${ }^{4}$ Pharmacology, Harbin Medical University; ${ }^{5}$ Department of \\ Urology Surgery, The Second Affiliated Hospital of Harbin Medical University, Harbin, Heilongjiang 150086, P.R. China
}

Received April 19, 2016; Accepted April 21, 2017

DOI: $10.3892 / \mathrm{ol} .2017 .6450$

\begin{abstract}
Inhibitor of growth family member 4 (ING4) is a candidate tumor suppressor that serves important roles in tumor growth and angiogenesis. In the present study ING4 expression was assessed in clear cell renal carcinoma (CCRC) tissues and its association with the progression of CCRC was determined. The expression of ING4 in 125 patients with CCRC was analyzed using reverse transcription-quantitative polymerase chain reaction (RT-qPCR), western blot analysis and immunohistochemical methods. A total of 40 adjacent normal renal tissues were used as control samples. The results identified that ING4 expression was positive in $100 \%$ of normal renal tissues, but in only $82.3 \%$ CCRC samples. RT-qPCR and western blotting results demonstrated that expression levels of ING4 mRNA and protein were significantly decreased in CCRC compared with in normal tissues $(\mathrm{P}<0.0001)$. ING4 expression was not associated with sex, age or tumor volume $(\mathrm{P}>0.05)$, but was associated with the nuclear grade of renal cancer $(\mathrm{P}<0.0001)$, the clinical stage of CCRC $(\mathrm{P}<0.0001)$ and lymphatic metastasis $(\mathrm{P}<0.0001)$. The results of the present study indicated that dysregulation of ING4 expression may be involved in the initiation and progression of CCRC.
\end{abstract}

\section{Introduction}

Inhibitor of growth (ING) is a family of tumor suppressor genes, and the well-known members of this family are inhibitor

Correspondence to: Dr Bai Zhao, Department of Urology Surgery, The Second Affiliated Hospital of Harbin Medical University, 246 Xuefu Road, Nangang, Harbin, Heilongjiang 150086, P.R. China E-mail: baizhao415@sina.com

Abbreviations: ING, inhibitor of growth; ING4, inhibitor of growth family member 4; CCRC, clear cell renal carcinoma; RT-qPCR, reverse transcription-quantitative polymerase chain reaction; NLS, nuclear localization signal

Key words: inhibitor of growth family member 4, clear cell renal carcinoma, tumor suppressor, clinical stage, lymphatic metastases of growth family member 1 (ING1), ING2, ING3, ING4 and ING5 $(1,2)$. The ING4 gene is localized at chromosome 12p13, consists of 8 exons and encodes a 29-kDa nuclear protein; its expression is ubiquitous in multiple different human tissues (3-5). The biological functions of ING4 have been extensively investigated, with a previous study demonstrating that ING4 protein promoted tumor protein p53 activity through direct interaction with it (6). As a consequence of the interaction between ING4 and p53, tumor protein p21 expression was upregulated, leading to cell cycle arrest $(7,8)$. In addition, ING4 altered apoptosis, contact inhibition and DNA repair $(9,10)$. ING4 has been considered as an important tumor suppressor gene, whose expression was significantly downregulated in a number of malignant tumors, including breast cancer, glioma and lung cancer $(8,11,12)$. However, the expression of ING4 has not been investigated in renal cell carcinoma. Approximately 15 million people worldwide are diagnosed with renal cell carcinoma annually (13), of which $75 \%$ of cases are clear cell renal carcinoma (CCRC) (14). The molecular mechanism of renal cell carcinoma has been extensively investigated, but there is no targeted therapy owing to a lack of targets. As ING4 was downregulated, or even mutated, in multiple cancer types $(8,15,16)$, In the present study, ING4 was inferred to be associated with multiple cancer types, potentially making it an ideal target for cancer therapy. In the present study, the level of ING4 mRNA and protein was probed in renal cancer tissue specimens in 125 patients with CCRC, and the adjacent normal tissue from 40 patients were used as a control.

\section{Materials and methods}

Patients and tissue specimens. Tumor tissue specimens were harvested from 125 patients with CCRC who underwent nephrectomy between October 2007 and September 2009 in the Department of Urology Surgery, The First, Second, and Fourth Affiliated Hospitals of Harbin Medical University (Heilongjiang, China). The 125 patients consisted of 79 males and 46 females with a median age of 52.5 years (range, 31-74 years). The average tumor diameter was 7.1 (range, 2.9-11.3 cm); 40 specimens of adjacent normal tissue were collected along with resection of the kidney to serve as controls. Neoplasm staging was performed according to the tumor-node-metastasis (TNM) classification of renal cell 
carcinoma (17). A total of 32 patients had stage I disease, 39 had stage II, 49 had stage III and 5 had stage IV. The nuclear grade of CCRC was as follows: 23 cases at grade I, 51 cases at grade II, 42 cases at grade III and 9 cases at grade IV. Tissue samples were divided into two types; one was stored at $-80^{\circ} \mathrm{C}$ following snap-freezing in liquid nitrogen and the other was fixed in $4 \%$ neutral formalin and embedded in paraffin. Informed written consent was obtained from the patients prior to tissue sample collection. The research protocol was approved by the Ethics Committee of Harbin Medical University.

Reverse transcription-quantitative polymerase chain reaction $(R T-q P C R)$. Total RNA was isolated using TRIzol reagent (Invitrogen; Thermo Fisher Scientific, Inc., Waltham, MA, USA). cDNA was reverse-transcribed from mRNA using the Reverse Transcription kit (Takara Biotechnology Co., Ltd., Dalian, China). cDNA samples were stored at $-20^{\circ} \mathrm{C}$ prior to use. A One-Step SYBR-Green I-based qPCR kit (PerkinElmer, Inc., Waltham, MA, USA) was used according to the manufacturer's protocol to detect ING4 mRNA levels in 40 CCRC tissue samples and corresponding normal renal tissues. The primer pairs for qPCR were as follows: ING4 forward, 5'-TCG TGCTCGTTCCAAAGG-3' and ING4 reverse, 5'-GGCAAT AGGTGGGTTCGTT-3'; $\beta$-actin forward, 5'-CCCAGCACA ATGAAGATCAAGATCAT-3' and $\beta$-actin reverse, 5'-ATC TGCTGGAAGGTGGACAGCGA-3'. The PCR program was as follows: Initial denaturation at $95^{\circ} \mathrm{C}$ for $10 \mathrm{sec}$, followed by 40 cycles of $95^{\circ} \mathrm{C}$ for $5 \mathrm{sec}$ and $53^{\circ} \mathrm{C}$ for $30 \mathrm{sec}$. There were three replicates of each PCR reaction. Results were quantified using the $2^{\Delta \Delta \mathrm{Cq}}$ method (18) following normalization to $\beta$-actin.

Western blotting. Western blotting was performed to detect the protein level of ING4 in the renal tissues obtained from the 40 patients. Tissue samples of $\sim 50 \mathrm{mg}$ were minced and ground in liquid nitrogen. The proteins were extracted using a Protein Extraction kit (Pierce; Thermo Fisher Scientific, Inc.), and the protein concentration was determined using the Bradford assay. A total of $100 \mu \mathrm{g}$ protein was separated by SDS-PAGE and transferred onto nitrocellulose membranes. The membranes were blocked with 5\% skimmed milk powder in PBS and subsequently incubated with rabbit anti-human ING4 polyclonal antibody (1:300; cat no. 40-7700; Invitrogen; Thermo Fisher Scientific, Inc.). Following washing, the membranes were incubated with horseradish peroxidase-conjugated goat anti-rabbit antibody (1:10,000; cat no. ZB-5301; Beijing Zhongshan Biotechnology Jinqiao Co., Ltd., Beijing, China). An enhanced chemiluminescence detection system (Shanghai Jiapeng Technology Co., Ltd., Shanghai, China) was used to visualize the protein bands. $\beta$-actin $(1: 1,000$; cat no. ab8226; Abcam, Cambridge, MA, USA) was used as an internal control. All experiments were repeated three times independently, and the level of ING4 protein expression was quantified using Scion imaging software 4.0 (Scion Corporation, Frederick, MD, USA).

Immunohistochemistry (IHC) analysis. IHC analysis was performed using a two-step immunohistochemical staining protocol as previously described (19). Briefly, 4- $\mu$ m-thick sections were blocked with $5 \%$ bovine serum albumin in PBS prior to incubation overnight at $4^{\circ} \mathrm{C}$ with rabbit anti-human
ING4 polyclonal antibody (1:100; cat no. 40-7700; Invitrogen; Thermo Fisher Scientific, Inc.). Following three washes with PBS containing Tween-20, the sections were then incubated with goat anti-rabbit secondary antibody (1:100; cat no. ZB-5301; Beijing Zhongshan Biotechnology Jinqiao Co., Ltd.) for $30 \mathrm{~min}$ at $37^{\circ} \mathrm{C}$. The tissues were visualized using 3,3'-diaminobenzidine tetrahydrochloride in water. Sections were counterstained using hematoxylin and sealed with neutral gum. Tissue sections incubated in PBS were used as negative controls and normal lung tissue, which was ING4-positive, was used as positive control. Cell membrane, cytoplasm and nucleus that were positively stained appeared as yellow-brown granules. The IHC results were quantified according to the number of positive cells and expressed as follows: $0 \%$, negative; $<25 \%,+; 25-50 \%,++; 51-75 \%,+++;>75 \%,++++$.

Statistical analysis. The densitometric analysis of western blotting and PCR results was performed using ImageJ software version 1.48 (National Institutes of Health, Bethesda, MD, USA). Comparison was performed using an unpaired Student's t-test. A non-parametric test was used to analyze the results from IHC staining and Spearman's rank correlation was used to analyze tissue positive for the expression of ING4. P<0.05 was considered to indicate a statistically significant difference.

\section{Results}

Decrease in ING4 mRNA in CCRC tissues. Initially, the level of ING4 mRNA expression was quantified in 40 paired tumor and adjacent normal tissues using RT-qPCR, with the data indicating that ING4 mRNA was downregulated in $100 \%$ (40/40) of CCRC tissues when compared with that in adjacent normal renal tissues $(0.4869 \pm 0.0448$ in CCRC vs. $0.7303 \pm 0.0434$ in normal tissue, $\mathrm{P}<0.0001$; Fig. 1$)$. The level of ING4 mRNA was positively associated with the nuclear grade of renal cancer $\left(r_{s}=-0.94076 ; P<0.0001\right)$, the clinical stage of CCRC $\left(r_{s}=-0.92400 ; P<0.0001\right)$ and lymphatic metastasis $\left(\mathrm{r}_{\mathrm{s}}=-0.78291 ; \mathrm{P}<0.0001\right)$; however, no association with patient sex or age, or the size of tumor was identified $(\mathrm{P}>0.05)$.

Decrease in ING4 protein expression in CCRC tissues. ING4 protein expression was evaluated by western blot analysis in the same 40 CCRC tissues and adjacent normal renal tissues. The expression of ING4 protein level in CCRC tissues was significantly decreased compared with in adjacent normal renal tissues $(0.4127 \pm 0.0723$ vs. $0.7488 \pm 0.0572$; $\mathrm{P}<0.0001$; Fig. 2$)$. The expression level of ING4 protein correlated with the nuclear grade of renal cancer $\left(r_{s}=-0.94655 ; \mathrm{P}<0.0001\right)$, the clinical stage of CCRC $\left(r_{s}=-0.90465, P<0.0001\right)$ and lymphatic metastasis $\left(r_{s}=-0.60608 ; \mathrm{P}<0.0001\right)$; however, no association between this expression and sex, age or tumor volume was identified $(\mathrm{P}>0.05)$.

Localization of ING4 in normal and CCRC tissue. All 40 normal renal tissue samples were positive for ING4 (100\%) in the renal tubular epithelial cell nuclei. ING4 protein expression was detected in all 125 CCRC samples. Of the 62 cases, ING4 was expressed in the nuclei of 11 cases, whereas in the remaining 51 cases, ING4 was mainly expressed in both cell membrane and cytoplasm (82.3\%, 51/62 cases) (Fig. 3B-F). 
A

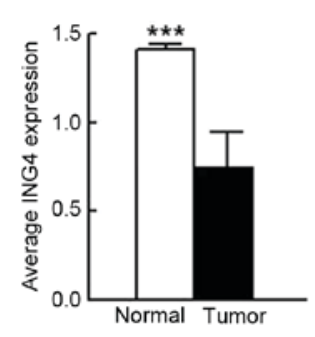

B

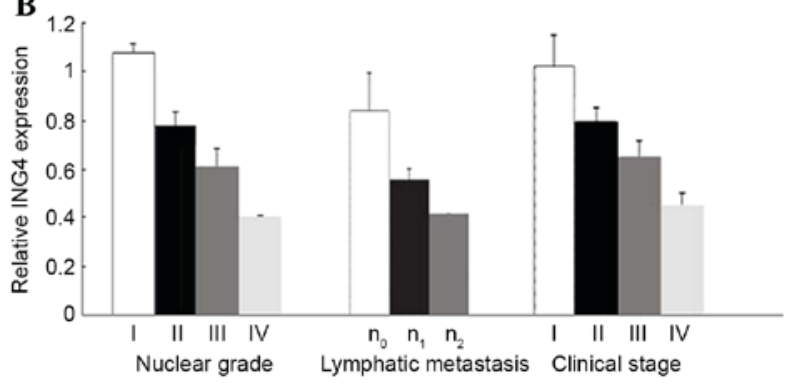

Figure 1. ING4 mRNA is significantly decreased in CCRC tissues when compared with normal renal tissues. (A) A total of 40 paired tumor and adjacent normal renal tissues were analyzed for ING4 mRNA level by reverse transcription-quantitative polymerase chain reaction. $\beta$-actin was used as an internal control. (B) Analysis of relative mRNA level (compared with normal renal tissue) according to Fuhrman nuclear grade (24) (grade I, n=7; grade II, n=16; grade 3, $n=13$; grade 4, $n=4)$, lymphatic metastasis $\left(n_{0}, n=29 ; n_{1}, n=7 ; n_{2}, n=4\right)$, tumor clinical stage (stage I, $n=9 ;$ stage II, $n=12 ;$ stage III, $n=14 ;$ stage IV, $\left.n=5\right)$. ${ }_{* * * *} \mathrm{P}<0.001$ by t-test. CCRC, clear cell renal carcinoma; ING4, inhibitor of growth family member 4.

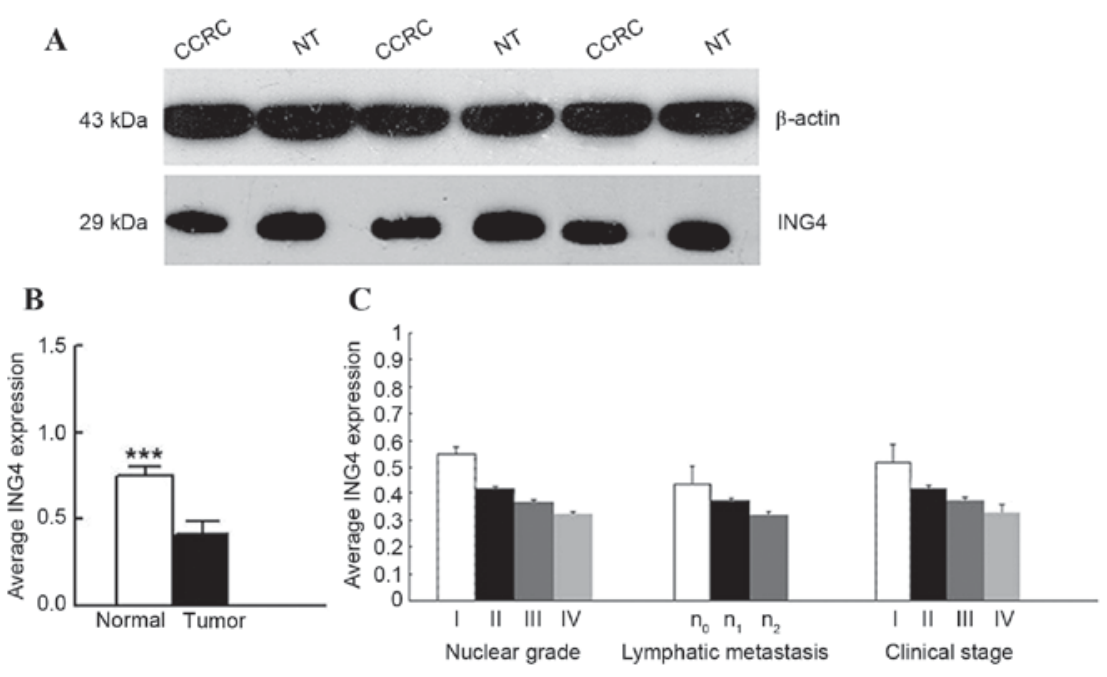

Figure 2. ING4 expression is significantly decreased in CCRC tissues when compared with normal renal tissues. (A) A total of 40 paired tumor and adjacent normal renal tissues were analyzed for ING4 expression using western blotting. Decreased ING4 expression was observed in CCRC tissues compared with adjacent normal renal tissues. $\beta$-actin was used as internal control. (B) Average ING4 expression in 40 paired tumor and normal renal tissues samples. (C) Analysis of the expression level of ING4 protein (compared with normal renal tissue) according to tumor nuclear grade (grade I, $\mathrm{n}=7$; grade II, $\mathrm{n}=16$; grade III, $n=13$; grade IV, $n=4$ ), lymphatic metastasis $\left(n_{0}, n=29 ; n_{1}, n=7 ; n_{2}, n=4\right)$, tumor clinical stage (stage I, $n=9$; stage II, $n=12$; stage III, $n=14 ;$ stage IV $\mathrm{n}=5) .{ }^{* * *} \mathrm{P}<0.001$ by t-test. CCRC, clear cell renal carcinoma; ING4, inhibitor of growth family member 4; NT, normal tissue.

A

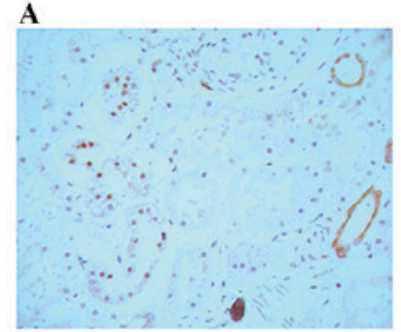

D

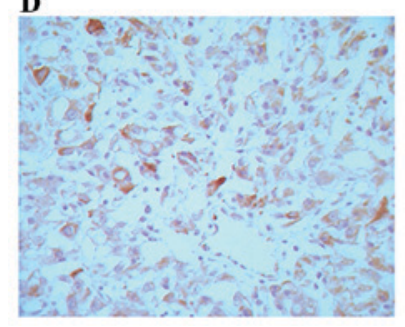

B

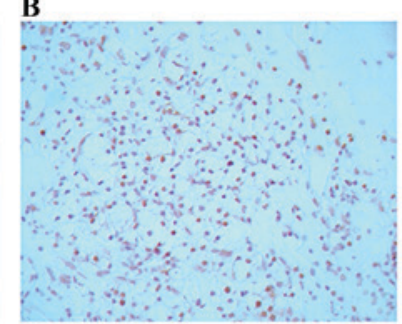

$\mathbf{E}$

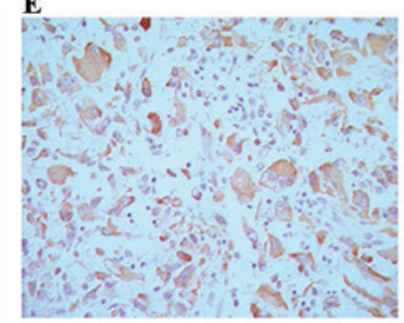

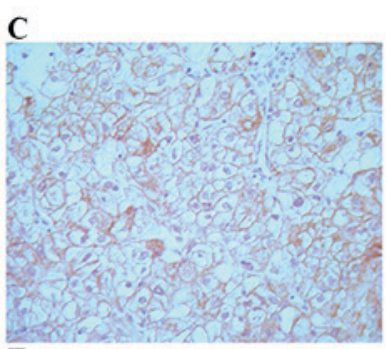

F

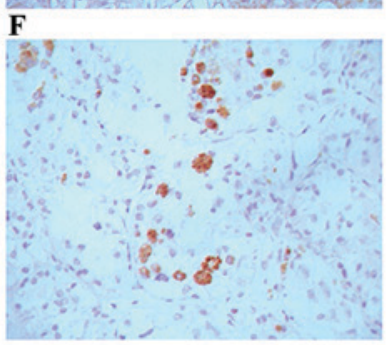

Figure 3. ING4 protein expression detected using the EnVision system in (A) normal renal tubular epithelial cell nuclei, and in renal cell carcinoma; (B) nuclear grade I, cellular expression; (C) nuclear grade II, cell membrane and cytoplasmic expression; (D) nuclear grade III, cytoplasmic expression; (E) nuclear grade IV, cytoplasmic expression; (F) positive expression of cytoplasmic granules. Magnification of all images, x400. ING4, inhibitor of growth family member 4 . 
Table I. ING4 protein in different nuclear grade renal cell carcinoma tissues.

\begin{tabular}{lcccrc}
\hline & & & \multicolumn{2}{c}{ ING4-positive $^{\text {a }}$} \\
\cline { 5 - 6 } Nuclear grade & Total cases, $n$ & ING4-negative & & + & ++ \\
\hline I & 34 & 7 & 6 & 10 & 11 \\
II & 39 & 15 & 9 & 7 & 8 \\
III & 29 & 21 & 6 & 1 & 1 \\
IV & 23 & 20 & 2 & 1 & 0 \\
\hline
\end{tabular}

${ }^{\mathrm{a}} \mathrm{P}<0.0001$ for ING4-positive vs. ING4-negative samples. ING4, inhibitor of growth family member 4.

Table II. ING4 protein expression in patients with clear cell renal carcinoma of the association between the clinical and pathological features.

\begin{tabular}{|c|c|c|c|c|}
\hline Clinical indicators & Patients, $\mathrm{n}$ & ING4-positive patients, $\mathrm{n}$ & ING4-positive expression, $\%$ & P-value \\
\hline \multicolumn{5}{|l|}{ Sex } \\
\hline Male & 79 & 40 & 50.63 & \multirow[t]{2}{*}{0.7621} \\
\hline Female & 46 & 22 & 47.83 & \\
\hline \multicolumn{5}{|l|}{ Age, years } \\
\hline$\leq 50$ & 41 & 19 & 46.34 & \multirow[t]{2}{*}{0.6107} \\
\hline$>50$ & 84 & 43 & 51.19 & \\
\hline \multicolumn{5}{|l|}{ Tumor size, cm } \\
\hline$\leq 7$ & 51 & 23 & 45.09 & \multirow[t]{2}{*}{0.0991} \\
\hline$>7$ & 74 & 39 & 52.70 & \\
\hline \multicolumn{5}{|l|}{ Staging grade } \\
\hline II & 27 & 12 & 44.44 & \multirow[t]{4}{*}{0.8463} \\
\hline II & 14 & 6 & 42.86 & \\
\hline III & 53 & 28 & 52.83 & \\
\hline IV & 31 & 16 & 51.61 & \\
\hline
\end{tabular}

ING4, inhibitor of growth family member 4.

The spatial expression of ING4 and the nuclear grade of CCRC is presented in Table I. The results indicated that ING4 protein expression was inversely associated with the nuclear grade of CCRC, and the rate of ING4 expression was increased with a low nuclear grade of CCRC $(\mathrm{P}<0.0001)$. No association was identified between ING4 expression and the sex, age, tumor size and clinical stage of the patients ( $\mathrm{P}>0.05$; Table II).

\section{Discussion}

ING is a family of tumor suppressor genes that includes ING1, ING2, ING3, ING4 and ING5. These genes participate in a number of cellular events, including the cell cycle, apoptosis and DNA repair. Overexpression of ING4 disrupted the cell cycle distribution of the cells, decreasing the number of cells in S phase (7). As a tumor suppressor gene, ING4 expression can suppress the growth of gliomas, breast tumors and squamous cell carcinomas of the head and neck $(4,5)$; however, its roles in CCRC remain unclear. In the present study, 125 clinical CCRC specimens underwent western blot analysis, RT-qPCR and IHC to probe ING4 expression in CCRC. The results of these analyses revealed that ING4 expression was significantly decreased in CCRC tissues compared with in the normal renal tissues at the mRNA and protein levels. ING4 was expressed in $100 \%$ of normal renal tissue samples (40/40 cases), but its expression was detectable only in $49.6 \%$ of CCRC samples (62/125). Garkavtsev et al (3) identified that the expression of ING4 mRNA in 50 glioma samples was significantly decreased compared with that in 5 adjacent brain tissues. Previous studies indicated that ING4 was downregulated in prostate cancer cell lines, melanoma, and in cancer of the stomach, liver, breast and lung $(15,16,20-22)$ and in $76 \%$ of the head and neck squamous cell carcinoma samples assessed (4). Taken together, the results of the present study indicated that a decrease in ING4 expression was associated with a variety of tumors, including CCRC.

Besides the dysregulation of ING4 expression, deletions and missense mutations in ING4 transcripts were detected in several tumor cell lines (4). Hybridization-mediated deletion of the ING4 locus was identified in between 10 and $20 \%$ of breast cancer cell lines and primary breast tumors, and in $66 \%$ 
of head and neck squamous carcinomas $(4,5)$. When deletions and missense mutations occurred in ING4 transcripts, the expression of ING4 decreased, leading to cell cycle dysregulation.

The results of the present study demonstrated that ING4 expression was not significantly associated with sex, age or tumor volume $(\mathrm{P}>0.05)$; however, ING4 expression was identified to be significantly associated with nuclear grade, clinical stage and lymphatic metastasis. ING4 expression decreased as the nuclear grade level increased $(\mathrm{P}=0.0038)$. These results were confirmed by RT-qPCR, western blot analysis and IHC. Therefore, downregulation of ING4 was associated with the progression and metastasis of CCRC. ING4 may cooperate with other genes to inhibit tumor cell growth in human renal cancer and may serve as a prognostic indicator for renal cancer.

In normal renal tissue, ING4 is expressed in the renal tubular epithelial cell nuclei. Of the 125 CCRC patient samples, 62 were positive for ING4 expression. In 51/62 ING4-positive samples $(82.3 \%)$, ING4 expression was observed outside nuclei in sites including the cell membrane and cytoplasm. The presence of membrane-bound ING4 has not yet been reported in other malignancies, indicating that the genetic changes of ING4 in the development of renal cell carcinoma may be distinct from those in other types of tumor. The nuclear localization signal (NLS) domain of ING4 is necessary for its interaction with p53 (19). Alternative splice variants of ING4, ING4_V2, ING4_V3 and ING4_V4 localized to the cytoplasm and lacked classical ING4 functions owing to a lack of NLS domain (23), suggesting that alternative splicing events may be one of the reasons for the cytoplasmic localization of ING4 in renal cancer.

Renal cell carcinoma does not typically respond to chemotherapy or radiation therapy, but responds well to immunotherapy (24). Therefore, it is important to develop novel therapeutic approaches for the treatment of CCRC. On the basis of the conclusions drawn from studies of other tumors, ING4 may serve as a valuable marker for CCRC, particularly the membrane-bound ING4 splicing variants, which may be targets for future immunotherapy strategies.

In conclusion, the results of the present study demonstrated that ING4 expression was downregulated in CCRC, and its expression was identified for the first time, to the best of our knowledge, to be associated with the advancement of clinical stage and nuclear grade level. Considering its roles in other types of tumor, ING4 may be a novel target for the diagnosis and therapy of CCRC.

\section{Acknowledgements}

The present study was partly supported by the Research Project of Heilongjiang Health Department (grant no. 2009-107), China.

\section{References}

1. Garkavtsev I, Kazarov A, Gudkov A and Riabowol K: Suppression of the novel growth inhibitor p33ING1 promotes neoplastic transformation. Nat Genetics 14: 415-420, 1996.

2. Garkavtsev I and Riabowol K: Extension of the replicative life span of human diploid fibroblasts by inhibition of the p33ING1 candidate tumor suppressor. Mol Cell Biol 17: 2014-2019, 1997.
3. Garkavtsev I, Kozin SV, Chernova O, Xu L, Winkler F, Brown E, Barnett GH and Jain RK: The candidate tumour suppressor protein ING4 regulates brain tumour growth and angiogenesis. Nature 428: 328-332, 2004

4. Gunduz M, Nagatsuka H, Demircan K, Gunduz E, Cengiz B, Ouchida M, Tsujigiwa H, Yamachika E, Fukushima K, Beder L, et al: Frequent deletion and down-regulation of ING4, a candidate tumor suppressor gene at $12 \mathrm{p} 13$, in head and neck squamous cell carcinomas. Gene 356: 109-117, 2005.

5. Kim S, Chin K, Gray JW and Bishop JM: A screen for genes that suppress loss of contact inhibition: Identification of ING4 as a candidate tumor suppressor gene in human cancer. Proc Natl Acad Sci USA 101: 16251-16256, 2004.

6. Guo Y, Meng X, Wang Q, Wang Y and Shang H: The ING4 binding with $\mathrm{p} 53$ and induced $\mathrm{p} 53$ acetylation were attenuated by human papillomavirus 16 E6. PLoS One 8: e71453, 2013.

7. Masayuki S, Makoto N, Pedeux RM, Kitahama-Shiseki M, Miura K, Okamura S, Onogi H, Higashimoto Y, Appella E, Yokota J and Harris CC: p29ING4 and p28ING5 bind to p53 and p300, and enhance p53 activity. Cancer Res 63: 2373-2378, 2003.

8. Liu E, Wu J, Cao W, Zhang J, Liu W, Jiang X and Zhang X: Curcumin induces $\mathrm{G} 2 / \mathrm{M}$ cell cycle arrest in a p53-dependent manner and upregulates ING4 expression in human glioma. J Neurooncol 85: 263-270, 2007.

9. Russell M, Berardi P, Wei G and Riabowol K: Grow-ING, Age-ING and Die-ING: ING proteins link cancer, senescence and apoptosis. Exp Cell Res 312: 951-961, 2006.

10. Nagashima M, Shiseki M, Pedeux RM, Okamura S Kitahama-Shiseki M, Miura K, Yokota J and Harris CC: A novel PHD-finger motif protein, p47ING3, modulates p53-mediated transcription, cell cycle control, and apoptosis. Oncogene 22: 343-350, 2003.

11. Tapia C, Zlobec I, Schneider S, Kilic E, Güth U, Bubendorf L and Kim S: Deletion of the inhibitor of growth 4 (ING4) tumor suppressor gene is prevalent in human epidermal growth factor 2 (HER2)-positive breast cancer. Hum Pathol 42: 983-990, 2011.

12. Wang QS, Li M, Zhang LY, Jin Y, Tong DD, Yu Y, Bai J, Huang Q, Liu FL, Liu A, et al: Down-regulation of ING4 is associated with initiation and progression of lung cancer. Histopathology 57: 271-281, 2010.

13. Organization WH, Ferlay J, Pisani P and Parkin DM: Globocan 2000: Cancer incidence, mortality and prevalence worldwide. Bray Freddie, 2001.

14. Zbar B, Klausner R and Linehan WM: Studying cancer families to identify kidney cancer genes. Annu Rev Med 54: 217-233, 2003.

15. Fang F, Luo LB, Tao YM, Wu F and Yang L: Decreased expression of inhibitor of growth 4 correlated with poor prognosis of hepatocellular carcinoma. Cancer Epidemiol Biomarkers Prev 18: 409-416, 2009.

16. Cai L, Li X, Zheng S, Wang Y, Li H, Yang J and Sun J: Inhibitor of growth 4 is involved in melanomagenesis and induces growth suppression and apoptosis in melanoma cell line M14. Melanoma Res 19: 1-7, 2009.

17. Edge SB and Compton CC: The American Joint Committee on Cancer: The 7th edition of the AJCC cancer staging manual and the future of TNM. Ann Surg Oncol 17: 1471-1474, 2010.

18. Livak KJ and Schmittgen TD: Analysis of relative gene expression data using real-time quantitative PCR and the 2(-Delta Delta C(T)) method. Methods 25: 402-408, 2001.

19. Zhao W, Yang L, Wang L, Zuo W, Yuan S, Yu J, Yu Q, Hu X, Wang S, Liu N, et al: Primary clear cell carcinoma of nasal cavity: Report of six cases and review of literature. Int J Clin Exp Med 7: 5469-5476, 2014.

20. Zhang X, Wang KS, Wang ZQ, Xu LS, Wang QW, Chen F, Wei DZ and Han ZG: Nuclear localization signal of ING4 plays a key role in its binding to p53. Biochem Biophys Res Commun 331: 1032-1038, 2005

21. Li M, Jin Y, Sun WJ, Yu Y, Bai J, Tong DD, Qi JP, Du JR, Geng JS, Huang $\mathrm{Q}$, et al: Reduced expression and novel splice variants of ING4 in human gastric adenocarcinoma. J Pathol 219: 87-95, 2009.

22. Yang HC, Sheng WH, Xie YF, Miao JC, Wei WX and Yang JC: In vitro and in vivo inhibitory effect of Ad-ING4 gene on proliferation of human prostate cancer PC-3 cells. Ai Zheng 28: 1149-1157, 2009 (In Chinese).

23. Raho G, Miranda C, Tamborini E, Pierotti MA and Greco A: Detection of novel mRNA splice variants of human ING4 tumor suppressor gene. Oncogene 26: 5247-5257, 2007.

24. Bleumer I, Oosterwijk E, De Mulder P and Mulders PF: Immunotherapy for renal cell carcinoma. Eur Urol 44: 65-75, 2003. 